\title{
EchoGéo
}

$10 \mid 2009$

La piraterie

\section{La diversité de la géographie en CPGE : une faiblesse ou un atout?}

\section{François Louveaux}

\section{OpenEdition}

1 Journals

\section{Édition électronique}

URL : https://journals.openedition.org/echogeo/11461

DOI : 10.4000/echogeo.11461

ISSN : 1963-1197

\section{Éditeur}

Pôle de recherche pour l'organisation et la diffusion de l'information géographique (CNRS UMR 8586)

Référence électronique

François Louveaux, «La diversité de la géographie en CPGE : une faiblesse ou un atout ? ", EchoGéo [En ligne], 10 | 2009, mis en ligne le 06 novembre 2009, consulté le 16 septembre 2021. URL : http:// journals.openedition.org/echogeo/11461; DOI : https://doi.org/10.4000/echogeo.11461

Ce document a été généré automatiquement le 16 septembre 2021.

EchoGéo est mis à disposition selon les termes de la licence Creative Commons Attribution - Pas d'Utilisation Commerciale - Pas de Modification 4.0 International (CC BY-NC-ND) 


\title{
La diversité de la géographie en CPGE : une faiblesse ou un atout?
}

\author{
François Louveaux
}

\section{La présence de la géographie en CPGE : plutôt discrète, peut-être stratégique}

1 Il y a 78000 étudiants en classes préparatoires aux grandes écoles (CPGE), un quart reçoit un enseignement de géographie. Placées à la charnière entre secondaire et supérieur, les trois grandes filières de CPGE - scientifique, économique et commerciale, littéraires - offrent une formation initiale de deux - voire trois - années, originale car pluri disciplinaire, organisée autour de la préparation des concours passés en fin de seconde année. Proches par leur organisation, les filières ont des effectifs inégaux 49000 en sciences, près de 20000 dans les classes économiques et commerciales, plus de 11000 en lettres (pour 740000 étudiants dans le cycle L de l'université). Elles différent aussi par leurs objectifs. Les étudiants des classes préparatoires scientifiques et économiques, intègrent en très grande majorité les grandes écoles auxquelles donnent accès les concours. Dans la filière littéraire, seule une minorité d'étudiants intègre effectivement de très rares grandes écoles. Les Ecoles Normales Supérieures, en fonction desquelles les enseignements sont organisés, recrutent un peu plus de 200 normaliens pour 4000 candidats; si l'on ajoute les débouchés offerts par les écoles de gestion, les Instituts d'Etudes Politiques, d'autres écoles ou filières universitaires sélectives, seul entre un quart et un tiers environ des étudiants littéraires intègre, les autres, en très grande majorité continuent leurs études à l'université, avec souvent la perspective de passer les concours de recrutement d'enseignants.

Dans le détail, chacune des filières se décline ensuite en voies différentes, offrant plus ou moins de débouchés directs, dans des écoles parfois inégalement cotées. Dans les CPGE Economiques et Commerciales, c'est le baccalauréat d'origine qui détermine la voie suivie - «scientifique » pour les bacheliers S, «économique " pour les bacheliers ES, « technologique » pour les bacheliers technologiques. Dans la filière scientifique, les 
classes BCPST (Biologie Chimie Physique Sciences de la Terre) constituent une voie originale - préparant aux écoles d'agronomie et vétérinaires. Les autres voies, majoritaires, reposent surtout sur mathématiques, physique, et, plus ou moins selon les sections, chimie et sciences de l'ingénieur. Là encore les étudiants se répartissent en fonction de leur baccalauréat d'origine - les bacs technologiques pour les sections dites TSI -, de leur niveau de résultats, de leurs affinités pour l'une des disciplines scientifiques, enfin de leurs projets d'intégration: les grandes écoles sont toujours généralistes, mais avec parfois une tonalité disciplinaire plus marquée et il y a entre elles un "prestige " supposé, conduisant à une hiérarchie subtile et qui est d'ailleurs l'un des points de friction majeur du système. Dans la filière littéraire, il y a des sections originales : la voie $\mathrm{BL}$, mathématiques, sciences sociales et lettres - qui recrute des bacheliers scientifiques -, la préparation à l'Ecole des Chartes, à l'Ecole spéciale Militaire. Pour les plus gros bataillons d'étudiants, la différence se fait en seconde année en fonction de l'Ecole Normale supérieure qui est visée et donc détermine les enseignements, soit l'ENS de Paris, soit l'ENSLSH à Lyon. Deux ENS, deux conceptions un peu différentes, des épreuves différentes - même si pour la première fois en 2009 se met en place une banque (partielle) d'épreuves - deux profils intellectuels un peu différents et surtout, pour ce qui nous concerne directement, une épreuve et donc un enseignement de géographie obligatoire dans la voie ENSLSH (pour l'ENS de Paris, c'est un enseignement de langue et culture de l'antiquité qui est obligatoire).

La géographie est inégalement présente dans les classes préparatoires. Absente des classes scientifiques, sauf pour la seconde année de BCPST, elle est discipline de base dans la voie "scientifique» de la filière Economique et Commerciale. Discipline obligatoire en première année des classes littéraires, elle est inégalement présente en seconde année. C'est un enseignement obligatoire pour tous à l'ENSLSH. Les " spécialistes » d'histoire-géographie des deux ENS - ceux qui choisissent de passer des épreuves de géographie aux concours-, suivent un enseignement spécifique. Elle n'est ainsi proposée qu'au quart des étudiants des CPGE. Les horaires d'enseignement sont très inégaux, le plus souvent réduits. Les thématiques sont très diverses, peuvent même paraître disparates. Pourtant ce sont près de 20000 étudiants qui reçoivent un enseignement de géographie dans les classes préparatoires, dont près d'un millier poursuivra ensuite des études de géographie et d'histoire à l'université, avec souvent pour objectif les concours de recrutement. Les classes préparatoires aux grandes écoles sont ainsi, statistiquement, un des lieux importants de l'enseignement de la géographie dans le supérieur. Au-delà, c'est dans ces classes que de futurs cadres et responsables se font une certaine idée de la géographie, ce qui n'est pas sans importance pour l'avenir de notre discipline.

\section{Diversité et cadre disciplinaire de l'enseignement de la géographie en CPGE}

4 La diversité des horaires n'a d'égale que celles des objets. En revanche les épreuves sont peu variées : dissertation pour presque tous, commentaire de documents, surtout de cartes, pour quelques-uns.

Dans des classes de formation initiale, originale car largement pluri disciplinaire, la géographie doit se situer ainsi par rapport au reste des disciplines enseignées dans chaque filière. Cette logique de filière explique la très grande diversité des horaires, des 
programmes. Cette diversité peut sembler faire de la géographie enseignée en CPGE une "discipline" hybride, sans vrai objet d'études, aux concepts fluctuants, aux méthodes strictement rhétoriques. Pourtant dans ces formations initiales, la géographie a des apports spécifiques : l' ancrage dans les sciences humaines et sociales - sans oublier que les espaces ont aussi des dimensions physiques -, dans le présent sans oublier qu'il ne se comprend que dans des temporalités emboitées -, la prise en compte de la complexité - celles des acteurs, des stratégies entre autres -, une approche multi scalaire et systémique.

6 Le cas de la filière BCPST est sans doute instructif. Jusqu'à la dernière réforme de ces classes, en 1996, la géographie occupait une place solide : un enseignement en première et en seconde année. Avec ce qui pouvait apparaître comme une certaine logique, l'enseignement privilégiait l'approche «biogéographique» et une approche économique- les types de productions ou de structures agricoles par exemple. Dans un souci d'alléger la charge de travail effectivement très lourde de ces étudiants, la solution « simple » retenue en 1996 est de supprimer la géographie et de gagner ainsi des heures précieuses pour des disciplines plus centrales. Il aura fallu une mobilisation de toute la communauté des géographes, de l'Inspection générale de l'Education nationale et jusqu'au Président de la République, pour que l'on décide en catastrophe de réintroduire un enseignement de géographie, seulement en seconde année, à raison d'une heure trente par semaine. L'intitulé de cet enseignement semble très flou aux géographes : "les territoires ruraux »... C'est pourtant le seul qui, à l'époque, a été trouvé pour convaincre les directeurs des écoles d'agronomie. Ceux-ci acceptent un enseignement de géographie, à condition qu'il porte sur un objet géographique et ne soit pas susceptible d'empiéter sur l'agronomie - ce qui leur paraît du ressort, ensuite, des Ecoles. Le terme de territoire signifie l'attention portée aux sociétés, dans toutes leurs dimensions, entre autres historiques, culturelles, politiques. Pour partie futurs aménageurs, les étudiants « d'agro » ont intérêt à penser qu'il n'y a jamais de solution efficace qui soit seulement technique ou économique.

\section{Un enseignement déterminé par les épreuves aux concours}

7 Le choix des épreuves conditionne largement les conditions d'enseignement. En géographie comme ailleurs, c'est la dissertation qui domine à l'écrit. Elle offre l'originalité relative d'obliger à construire les analyses à partir de faits précis, mesurés, localisés, mis en relation, à montrer l'emboîtement des échelles, la diversité significative des situations, la prise en compte de phénomènes de natures différentes mais combinés dans des systèmes complexes et évolutifs. On demande souvent de réaliser croquis, cartes, tableaux, organigrammes: ils ne doivent pas être des décorations, mais des supports d'analyses précises, des outils de raisonnement, de comparaison. Cet objectif est très inégalement atteint.

Plus encore que les épreuves, les thèmes mis au programme des concours comme les sujets de composition sont de puissants outils d'évolution des pratiques pédagogiques. Les préparateurs s'ajustent, pour le plus grand bénéfice des étudiants qui leur sont confiés, aux demandes des jurys, quasi exclusivement composés d'universitaires, d'enseignants du supérieur. Pour les concours des Ecoles Normales Supérieures, le programme est défini chaque année, avec une alternance entre une question 
thématique et une question régionalisée - comme l'indique le tableau 1 qui reprend les thèmes mis aux programmes ces dernières années. Les thèmes sont variés. Ils tâchent de coller à la production scientifique - ce qui permet aux étudiants d'avoir accès à des ouvrages universitaires. Ils correspondent souvent à des lieux ou des questions mis en avant par l'actualité : on est loin de la géographie « froide » qui serait, dit-on, enseignée en classes préparatoires. On mesure la part de risque que comportent de tels choix, mais les avantages sont bien supérieurs. Au-delà des thèmes d'études, les sujets précis sont des signaux forts émis vers les étudiants et les préparateurs. Dans le concours de l'ENSLSH, la dissertation de géographie est une épreuve « commune ", non réservée aux seuls historiens-géographes. Les sujets récents sont pourtant tous construits autour de concepts clés de la géographie - territoires/réseaux et flux/mobilités pour reprendre les trois dernières années. On mesure bien l'ambition du jury et ses exigences vis-à-vis des candidats et donc des préparateurs. Si les $9 / 10^{\mathrm{e}}$ des candidats ne feront plus de géographie ensuite, le concours est l'occasion d'approfondir un thème, d'étudier un ensemble spatial, d'évoquer toujours des lieux et des liens, mais aussi de faire comprendre ce qu'est la géographie, à travers ses objets, ses concepts, ses méthodes. On est loin du simple exercice de rhétorique générale. On est bien dans la logique de l'interdisciplinarité qui n'a de sens que si chaque discipline joue sa propre partition, apporte son propre angle de vue, ce qui rend alors fructueuse la confrontation des regards.

Une épreuve dite de "commentaire de documents " s'ajoute à la dissertation pour certains étudiants, essentiellement ceux qui destinent à l'histoire et à la géographie. Elle consiste en un commentaire de carte topographique française au $1 / 50000^{\mathrm{e}}$ ou au $1 / 25000^{\circ}$, accompagnée de documents annexes - autre édition de la carte, autre échelle, photographies, statistiques, texte littéraire ou scientifique. On retrouve l'exercice canonique, le passage longtemps obligé de tous les géographes et historiens dès la première année de Licence, et jusque récemment l'épreuve reine du CAPES et de l'agrégation. L'attachement à l'exercice n'a pas empêché des évolutions et d'abord, hautement symbolique, la disparition toute récente - officielle depuis un an - du célébrissime "carton géologique simplifié ». L'adjonction de documents annexes bien choisis permet de sortir partiellement de l'ambigüité de cette épreuve.

Ce tableau des épreuves est révélateur. La géographie enseignée en classe préparatoire est très classique dans ses formes. Elle peut être très actuelle dans ses thèmes et problématiques, lesquelles dépendent des universitaires, pour l'essentiel. Les épreuves de concours ne résument pas la totalité de ce qui est enseigné, entre autres dans les classes de première année littéraire où la géographie est d'abord discipline de formation générale pour des étudiants qui pour la moitié d'entre eux n'en feront plus ensuite. Ici la liberté, la responsabilité des enseignants est plus grande, avec le triple objectif de préparer aux exigences des concours, de montrer ce que peut être une analyse géographique, de s'intégrer dans une démarche multi disciplinaire. Les supports sont très variés - la France est souvent étudiée - les angles d'approche doivent être multiples, avec la volonté - désormais commune à toutes les disciplines enseignées en CPGE - d'ouvrir aux démarches de recherche, d'initier au travail personnel et au travail en équipe. Cela correspond au cahier des charges clairement défini lors de la refonte des classes préparatoires littéraires engagée depuis 2007. 


\section{Les évolutions nécessaires : décloisonner}

11 Au-delà d'une apparente, rassurante ou inquiétante stabilité, la géographie en CPGE doit évoluer. Alors que ces classes sont volontiers présentées comme des bastions - à prendre ou à défendre - elles sont étroitement dépendantes. En amont, la réforme du lycée risque de bouleverser radicalement les apprentissages des futurs préparationnaires. En aval, la réforme en cours des concours de recrutement, l'horizon non avoué mais très clair des étudiants des CPGE littéraires, modifiera dans les classes préparatoires comme à l'université, les attentes, les formations. Les évolutions passent par une réflexion sur la nature des épreuves, sur les contenus d'enseignement, sur les rapports à établir entre classes préparatoires et universités, rapports institutionnels sans doute, rapports individuels, personnels plus sûrement encore. Les propos qui suivent ne sont ni collectifs, ni consensuels. Ils se veulent simplement des introductions à des débats sans doute nécessaires. Ils n'ont pas la prétention de proposer des solutions adaptées à toutes les filières.

12 Pour modifier éventuellement les épreuves des concours, deux voies se proposent $a$ priori. La première est celle $\mathrm{du}$ commentaire de dossier documentaire, épreuve largement présente dans les concours d'enseignement, de recrutement et dans la promotion interne. L'autre piste, serait d'imaginer une épreuve faisant appel aux Technologies Usuelles de la Communication et de l'Information. La difficulté serait peut-être de faire accepter l'idée " d'abandonner la dissertation », l'épreuve noble, et donc de risquer d'apparaître aux yeux de étudiants ou des collègues comme une discipline moins «noble ». Ne serait-il pas temps aussi de considérer que les concours devraient évaluer plusieurs types de compétences et pas uniquement celles requises pour la dissertation, surtout si l'on prétend diversifier les formes d'excellence et si l'on affirme qu'excellences et réussites se pensent au pluriel?

Il est évident en tous cas que, par le biais d'une épreuve ou non, l'utilisation des outils informatiques en géographie doit entrer dans nos classes. Il y va de l'intérêt bien compris de nos étudiants qui, une fois en master, ont des difficultés car ils ne connaissent même pas, et maîtrisent encore moins des outils indispensables. Il y va aussi de l'image et de la réalité de notre discipline. La géographie ne se fait plus seulement avec des crayons de couleurs, des cartes topographiques et du papier calque. Les objets étudiés, les champs, les sources, les méthodes de traitement se sont démultipliées, et les classes préparatoires n'en disent pas grand-chose. Electronique et informatique ne sont pas seulement des outils au service d'une démarche géographique qui resterait "classique ", mais ils permettent de construire des savoirs, de créer des connaissances. Les géographes acquièrent avec la maîtrise de ces outils, les SIG en particulier, une vraie reconnaissance auprès d'autres chercheurs, ou d'employeurs. Pourquoi la géographie n'assumerait-elle pas son caractère original dans des études «littéraires" en assumant cette dimension "technique» sans que ce terme n'apparaisse nécessairement péjoratif?

Que l'on se rassure, il y a déjà des moyens d'initier nos étudiants à ces méthodes et démarches. Les interrogations orales, "colles", peuvent être l'occasion de présenter une courte synthèse faite en solo ou à plusieurs, sur un aspect des thèmes mis au programme, à partir d'une recherche bibliographique. En seconde année, entre l'écrit et l'oral -une période où les emplois du temps sont un peu moins contraints, on peut proposer des stages d'initiation aux outils informatiques appliqués à la géographie- par 
exemple, méthodes statistiques, cartographie, SIG. Le plus riche est d'ailleurs de faire ses mini stages avec l'université proche - en convention - ce qui a aussi l'avantage de créer des rencontres fécondes entre enseignants chercheurs et étudiants de CPGE, de faire connaitre aux étudiants des lieux qu'ils fréquenteront, de faire tomber bien des préjugés.

On l'aura compris la géographie enseignée en CPGE doit évoluer et c'est pour elle une chance. Cette évolution ne peut se faire sans être étroitement liée aux évolutions à l'université. Avec les thèmes choisis pour les programmes, les sujets posés dans les concours, on peut facilement faire percoler en CPGE les nouvelles thématiques de recherche, des concepts rénovés - tout en tenant compte des contraintes liées à un public d'étudiants non spécialistes et de niveau bac plus deux. Cela nécessite bien sûr un dialogue avec les professeurs de CPGE - et l'Inspection générale peut y tenir une grande place - pour vérifier que ce qui est proposé est faisable et aussi en accord avec une géographie qui reste discipline de formation générale initiale pour non spécialistes. La question de l'épreuve ou des épreuves - écrites et orales- destinées aux futurs spécialistes de géographie et/ou histoire est un peu plus complexe. La situation actuelle n'est pas totalement satisfaisante, la mise au point d'épreuves nouvelles délicates à la fois sur le plan technique et sur le plan symbolique - une dimension "considérable " dans les classes préparatoires, à la fois une richesse et un frein.

Tout cela suppose des rencontres entre universitaires et enseignants de CPGE, pour le plus grand bien de la discipline mais aussi, mais surtout des étudiants qui nous sont confiés et qui, pour la plupart iront ensuite à l'université, entre autres pour préparer les concours d'enseignement. Aujourd'hui les nouveaux enseignants recrutés en CPGE sont de plus en plus souvent docteurs, ont reçu la même formation que leurs camarades qui sont devenus enseignants-chercheurs. Les géographes constituent une petite communauté, il est possible de se rencontrer et de se connaître. Il nous est sans doute ainsi plus facile de dépasser entre CPGE et universités des préjugés qui ne sont pas sans explications, mais conduiraient à négliger l'intérêt de nos étudiants et de notre discipline.

\section{RÉSUMÉS}

Discipline de concours, discipline de formation générale dans un cadre pluridisciplinaire, formation initiale pour de futurs étudiants et enseignants historiens-géographes, la géographie en classes préparatoires doit être tout cela à la fois. Cette diversité est-elle une faiblesse ou, aussi, une richesse possible, pour peu que l'on sache articuler enseignement en CPGE et à l'université, en pleine connaissance et reconnaissance réciproques, loin des clichés? 
AUTEUR

FRANÇOIS LOUVEAUX

François Louveaux (francois.louveaux@wanadoo.fr) est professeur en classes préparatoires. 